\title{
Pricing European Put Option in a Geometric Brownian Motion Stochastic Volatility Model
}

\author{
Kolawole Imole Oluwakemi ${ }^{1}$, Mataramvura Sure ${ }^{2}$, Ogunlade Temitope Olu ${ }^{3}$ \\ ${ }^{1}$ School of Mathematical and Computer Sciences, Heriot-Watt University, Edinburgh, Scotland \\ ${ }^{2}$ Department of Mathematical Sciences, University of Cape Town, Cape Town, South Africa \\ ${ }^{3}$ Department of Mathematics, Ekiti State University, Ado-Ekiti, Nigeria
}

Email address:

topsmatic@gmail.com (O. T. Olu)

\section{To cite this article:}

Kolawole Imole Oluwakemi, Mataramvura Sure, Ogunlade Temitope Olu. Pricing European Put Option in a Geometric Brownian Motion Stochastic Volatility Model. Applied and Computational Mathematics. Vol. 6, No. 5, 2017, pp. 215-221. doi: 10.11648/j.acm.20170605.11

Received: June 9, 2017; Accepted: June 26, 2017; Published: September 7, 2017

\begin{abstract}
Stochastic volatility models were introduced because option prices have been mis-priced using Black-Scholes model. In this work, focus is made on pricing European put option in a Geometric Brownian Motion (GBM) stochastic volatility model with uncorrelated stock and volatility. The option is priced using two numerical methods (Crank-Nicolson and Alternating Direction Implicit (ADI) finite difference). Numerical schemes were considered because the closed form solution to the model could not be obtained. The change in option value due to changes in volatility, maturity time and market price of volatility risk are considered and comparison between the efficiency of the numerical methods by computing the CPU time was made.
\end{abstract}

Keywords: Geometric Brownian Motion (GBM), Alternating Direction Implicit (ADI) Scheme Crank Nicolson Scheme, Black-Scholes Model, European Put Option

\section{Introduction}

Black-Scholes model is theoretically appealing and it has been embraced by many investors. However, after carrying out some empirical studies on the model, it has been revealed that the assumption of constant volatility is not sufficient in specifying the return on stock. Volatility itself is observed to have some variability and stochastic volatility models have been proposed to model this variability [8]. One of the proposed stochastic volatility models is Geometric Brownian motion (GBM). This model supports the limited liability of shareholders which means that the worse that can happen to a shareholder of a company going bankrupt is to lose their investment and they cannot be held responsible for the company's debt. It is noted that GBM has a great advantage of the simplicity [1]. As opposed to the first model for stock price dynamics postulated by Bachelier in 1900, which had the change in the stock price itself proportional to a Brownian motion increment, since Brownian motion can take negative values, it then implies the stock can become negative [11]. The main source of randomness of a financial derivative in Black-Scholes model is the Brownian motion in the underlying asset, which is tradable. This means that any option can be hedged with the underlying and a risk-free asset, hence the model is said to be complete. However, stochastic volatility models are incomplete because there is an additional source of randomness (i.e. another Brownian motion in the volatility model.) that is not tradable, hence contingent claims (such as European options) cannot be priced by the no arbitrage principle.

Due to the complexity of some models whose closed form solution does not exist, Finite Difference Method (FDM) has been employed in the valuation of options. And in instances where closed form solution exists, they seem difficult to compute and hence approximate methods are being considered appropriate [3]. The idea behind approximating partial differential equations using FDM is to approximate the derivatives using divided difference alongside some specified boundary conditions, these procedure transforms the PDE into an ordinary differential equation, this is called spatial discretization. Solving the evolving ordinary differential equation which is a function of time $t$, requires initial condition and it is called time discretization. 


\section{Dynamics of GBM Stochastic Volatility Model}

Consider the following GBM stochastic volatility model representing the dynamics of a stock and its volatility under a subjective measure $\mathbb{P}$

$$
\begin{gathered}
d S(t)=\alpha S(t) d t+\sigma(t) S(t) d B_{1}(t) \\
d \sigma(t)=k \sigma(t) d t+\theta \sigma(t) S(t) d B_{2}(t)
\end{gathered}
$$

Where $B_{1}(t)$ and $B_{2}(t)$ are non correlated standard independent Brownian motions defined on the probability space $\left(\Omega, \mathcal{F}, \mathcal{F}_{t}, \mathbb{P}\right)$. The model (1)-(2) under consideration is called Geometric Brownian motion because the logarithm of the underlying $S(t)$ and $\sigma(t)$ follows Brownian motion respectively. To make complete the model (1)-(2) means there is need to first specify the market price for risk, which reflects the expected excess return per unit risk over the riskfree rate [7]. The market price for risk is the bridge inbetween investors risk averting abilities. Having introduced the market price of risk, it is therefore sufficient that we apply a Girsanov change of probability measure theorem which leads to a martingale measure.

In (1) we see that $\alpha$ is the risky rate of return which depends on individual investors, hence by the concept of change of measure we want a risk-free rate of return $r$, this can be achieved by removing the risk from the drift.

Theorem. (Girsanov Theorem [10]) Let $B(t)$ for $t \in$ $[0 ; T]$ be independents Brownian motion on a given probability space $\left(\Omega, \mathcal{F}, \mathcal{F}_{t}, \mathbb{P}\right)$. where $\mathcal{F}_{t}$ is a filtration on this Brownian motion. Let $\psi(t)$ be an adapted process

$$
\begin{gathered}
B^{*}(t)=B(t)+\int_{0}^{t} \psi(t) d s \\
Z(t)=\exp \left(-\frac{1}{2} \int_{0}^{t} \psi(s)^{2} d s-\int_{0}^{t} \psi(t) d B(s)\right)
\end{gathered}
$$

Where $Z(t)=\frac{d \mathbb{P}^{*}}{d \mathbb{P}}$ is the change in the probability measure called the Radon-Nikodym derivative. The new probability measure is given by,

$$
\mathbb{P}^{*}=\int_{A} Z(t) d \mathbb{P} \forall A \in \mathcal{F}
$$

The process $B^{*}(t)$ is a Brownian motion under the equivalent martingale measure $\mathbb{P}^{*}$, it has mean zero and variance $t$.

Applying the Girsanov change of probability measure theorem to (1)-(2), the equivalent Brownian motions under the new probability measure are as follow:

$$
\begin{gathered}
B_{1}^{*}(t)=B_{1}(t)+\frac{\alpha-r}{\sigma} t \\
B_{2}^{*}(t)=B_{2}(t)+\gamma t
\end{gathered}
$$

According to [9], the process, $\gamma t=\frac{\lambda(S, \sigma, t)}{\theta}$ where $\lambda(S, \sigma, t)$ is the market price of volatility risk and $\theta$ is the volatility of volatility.

The change of probability measure $\frac{d \mathbb{P}^{*}}{d \mathbb{P}}$ is given by

$$
Z(t)=\exp \left(-\frac{1}{2} \int_{0}^{t}\left(\psi_{1}^{2}(s)+\psi_{2}^{2}(s)\right) d s-\int_{0}^{T} \psi_{2}(s) d B_{1}(s)-\int_{0}^{T} \psi_{2}(s) d B_{2}(s)\right)
$$

Where $\psi_{1}(s)=\frac{\alpha-r}{\sigma} t$ and $\psi_{2}(s)=\gamma t$

The term $\lambda(S, \sigma, t)$ is called the risk premium factor or the market price of volatility, while $\frac{\alpha-r}{\sigma}$ is stock market price of risk. Let $\lambda=0$ and making appropriate substitution for $B_{1}^{*}(t)$ and $B_{2}^{*}(t)$, the model (1) and (2) in the risk neutral world is

$$
\begin{aligned}
& d S=r S d t+\sigma S d B_{1}^{*}(t) \\
& d \sigma=k \sigma d t+\theta \sigma d B_{2}^{*}(t)
\end{aligned}
$$

\subsection{GBM Stochastic Volatility PDE}

In this section, derivation of the PDE for pricing contingent claims in a GBM stochastic volatility model (5)(6) was made.

Portfolio Dynamics. Let us consider a port folio $\Pi$ consisting of lunit of option $V, \phi$ units of option $U$ and $\Delta$ units of the underlying asset $S$ given by

$$
d V=\frac{\partial V}{\partial t} d t+\frac{\partial V}{\partial S}\left(r S d t+\sigma S d B_{1}^{*}(t)\right)+\frac{\partial V}{\partial \sigma}\left(k \sigma d t+\theta \sigma B_{2}^{*}(t)\right)+\frac{1}{2} \frac{\partial^{2} V}{\partial S^{2}}\left(\sigma^{2} S^{2} d t\right)+\frac{1}{2} \frac{\partial^{2} V}{\partial \sigma^{2}}\left(\sigma^{2} \theta^{2} d t\right)
$$

Similarly, the option $U$ gives the following PDE

$$
d U=\frac{\partial U}{\partial t} d t+\frac{\partial U}{\partial S}\left(r S d t+\sigma S d B_{1}^{*}(t)\right)+\frac{\partial U}{\partial \sigma}\left(k \sigma d t+\theta \sigma B_{2}^{*}(t)\right)+\frac{1}{2} \frac{\partial^{2} U}{\partial S^{2}}\left(\sigma^{2} S^{2} d t\right)+\frac{1}{2} \frac{\partial^{2} U}{\partial \sigma^{2}}\left(\sigma^{2} \theta^{2} d t\right)
$$




\subsection{Riskless Property of the Portfolio}

For the portfolio to be hedge-able against the fluctuation in stock and volatility, this directly implies that the random terms must be equal to zero, we then have the following;

$$
\begin{gathered}
\sigma S \frac{\partial V}{\partial S}+\Delta \sigma S+\phi \sigma S \frac{\partial U}{\partial S}=0 \\
\theta \sigma \frac{\partial V}{\partial \sigma}+\phi \sigma \theta \frac{\partial U}{\partial \sigma}=0
\end{gathered}
$$

The riskless change in portfolio $\mathrm{d}_{t}$ after substituting the hedge parameter Delta is

$$
\mathrm{d} \Pi_{t}=(A+\phi B) d t
$$

\subsection{Risk-Free Rate Earned by the Portfolio}

In the risk-neutral world, the expected return on all assets in the absence of arbitrage opportunities is the risk-free rate $r$. Therefore the portfolio (7) must earn a risk free rate $r$, (i.e. the change in portfolio $\mathrm{d} \Pi_{t}=r \Pi_{t} d t$ ).

$$
\mathrm{d} \Pi_{t}=r(V+\Delta S+\phi U) d t
$$

Equating the two expression we have for the change in portfolio (13) and (14) to each other

$$
A+\phi B=r V-r S \frac{\partial V}{\partial S}-r S \phi \frac{\partial U}{\partial S}+r \phi U
$$

get

$$
\frac{A-r V-r S \frac{\partial V}{\partial S}}{\frac{\partial V}{\partial \sigma}}=\frac{B-r U+r S \frac{\partial U}{\partial S}}{\frac{\partial U}{\partial \sigma}}
$$

The left-hand side of (16) is a function of $\mathrm{V}$ only, so also the right-hand side is a function of $U$ only. If the equation above holds for any put option of any maturity and strike price, then the left-hand side and right-hand side must be independent from the type of option being considered. Hence it indicates that each side is equal to $\lambda(S, \sigma, t)$, the volatility risk premium which indicates that we are faced with an incomplete market [7].

$$
\frac{A-r V-r S \frac{\partial V}{\partial S}}{\frac{\partial V}{\partial \sigma}}=\lambda(S, \sigma, t)
$$

Substituting the risk premium $\lambda \sigma$ that the volatility premium is proportional to the volatility, we have

$$
A-r V-r S \frac{\partial V}{\partial S}=\lambda \sigma \frac{\partial V}{\partial \sigma}
$$

Substituting $A$ and $E^{2}$ into the above, gives us the partial differential equation for the model (1)-(2) under the equivalent martingale probability measure $\mathbb{P}^{*}$ in the riskneutral world as

Making necessary simplification and factorization in (15) we

$$
\frac{\partial V}{\partial t}+r S \frac{\partial V}{\partial S}+E \sigma \frac{\partial V}{\partial \sigma}+\frac{1}{2}\left(\sigma^{2} S^{2}\right) \frac{\partial^{2} V}{\partial S^{2}}+\frac{1}{2}\left(\sigma^{2} \theta^{2}\right) \frac{\partial^{2} V}{\partial \sigma^{2}}-r V=0
$$

\section{Finite Difference Method (FDM)}

FDM has been employed in the valuation of options due to the complexity of some models whose closed form solution does not exist. And in instances where closed form solution exist, they seem difficult to compute and hence approximate methods are being considered appropriate [3].

Having realized that the model (1)-(2) is not affine, and the closed form solution to such models rarely do exist, we therefore resort to numerical methods in solving (18) above.

We consider Crank-Nicolson and Alternating Direction Implicit (ADI) finite difference methods in approximating the PDE (18).

\subsection{Crank-Nicolson Scheme}

This numerical scheme is a $\theta$ scheme of the implicit type for which $\theta=\frac{1}{2}$. It is centered in space and in time. It is unconditionally stable and convergent [2]. Crank-Nicholson FDM is the weighted average of both the implicit and explicit finite difference methods.

Consider four boundary conditions, these conditions are based on the principle supporting European put option (i.e.the right is claimed only if the strike price $\mathrm{K}$ is greater than the stock price at maturity). The boundary conditions attached to the stock are the following;

At $x=0$ Dirichlet boundary condition [6]. $V(0, y, \tau)=$ $K e^{-r t}$

At $x=x_{\max }[12], \lim _{x \rightarrow \infty} V(x, y, \tau)=0$

Note that $\infty$ in our case is $x_{\text {max }}$. This condition is realistic in the sense that if the stock price is at its maximum, the owner of a put option need not to exercise it.The aforementioned statement is valid because the maximum stock price are chosen to be greater than the strike price $\left(x_{\max }>K\right)$ for option pricing domain.

The boundary conditions attached to the volatility are as follows;

At $y=0$ Dirichlet boundary condition

$\lim _{y \rightarrow \infty} V(x, y, \tau)=\max \left(K e^{-r t}-x, 0\right)$

At $\mathrm{y}=y_{\max }$ Neumann boundary condition [6] $\lim _{y \rightarrow \infty} \frac{\partial V}{\partial y}(x, y, \tau)=0$

Replacing $S$ by $x, \sigma$ by $y$ and $t$ by $\tau$, we write the PDE (18) as

$$
\frac{\partial V}{\partial \tau}-r x \frac{\partial V}{\partial x}-E y \frac{\partial V}{\partial y}-\frac{1}{2}\left(\sigma^{2} x^{2}\right) \frac{\partial^{2} V}{\partial x^{2}}-\frac{1}{2}\left(\sigma^{2} \theta^{2}\right) \frac{\partial^{2} V}{\partial y^{2}}+r V=0
$$




$$
\begin{gathered}
\frac{\partial V}{\partial t}=A V \\
A V=r x \frac{\partial V}{\partial x}+E y \frac{\partial V}{\partial y}+\frac{1}{2}\left(\sigma^{2} x^{2}\right) \frac{\partial^{2} V}{\partial x^{2}}+\frac{1}{2}\left(\sigma^{2} \theta^{2}\right) \frac{\partial^{2} V}{\partial y^{2}}-r V=0
\end{gathered}
$$

For the grid, let the number of time steps be $L$, and the number of internal nodes on $x$-axis $=\mathrm{M}, y$-axis $=\mathrm{N}$ respectively. All having equally spaced interval by $\Delta \tau=\frac{T}{L}$, $\Delta x=\frac{x_{\max }}{M}, \Delta y=\frac{y_{\max }}{N}$. We make use of the approximatio $V_{i, j} \approx V\left(x_{i}, y_{i}\right)(i=0, \ldots M+1, j=0, \ldots N+1)$

The points $x_{M+1}$ and $y_{N+1}$ are the maximum stock price and volatility respectively.

The two spaces to be discretized are stock price and volatility, we will make use of the second order central finite difference formula

$$
A V=a_{i, j} V_{i, j-1}+b_{i, j} V_{i, j}+c_{i, j} V_{i, j+1}+d_{i, j} V_{i-1, j}+e_{i, j} V_{i+1, j}
$$

Where

$$
\left\{\begin{array}{c}
a_{i, j}=-\frac{E y_{j}}{2 \Delta y}+\frac{y_{j}^{2} \theta^{2}}{2(\Delta y)^{2}} \\
b_{i, j}=-\frac{x_{i}^{2} y_{j}^{2}}{(\Delta x)^{2}}-\frac{y_{j}^{2} \theta^{2}}{(\Delta y)^{2}}-r \\
c_{i, j}=\frac{E y_{j}}{2 \Delta y}+\frac{y_{j}^{2} \theta^{2}}{2(\Delta y)^{2}} \\
d_{i, j}=-\frac{r x_{i}}{2 \Delta x}+\frac{x_{i}^{2} y_{j}^{2}}{(\Delta x)^{2}} \\
e_{i, j}=\frac{r x_{i}}{2 \Delta x}+\frac{x_{i}^{2} y_{j}^{2}}{(\Delta x)^{2}}
\end{array}\right.
$$

Putting these together, the PDE can be written as

$$
\frac{\partial V}{\partial \tau}=a_{i, j} V_{i, j-1}+b_{i, j} V_{i, j}+c_{i, j} V_{i, j+1}+d_{i, j} V_{i-1, j}+e_{i, j} V_{i+1, j}
$$

To get the system of equation from this difference equation, we typically pick a point from the stock and run It through for each point of the volatility. When $i=1$, we run through $j=1, \ldots N$

$$
\begin{gathered}
\frac{\partial V_{11}}{\partial \tau}=a_{11} V_{10}+b_{11} V_{11}+c_{11} V_{12}+d_{11} V_{01}+e_{11} V_{21} \\
\frac{\partial V_{12}}{\partial \tau}=a_{12} V_{11}+b_{12} V_{12}+c_{12} V_{13}+d_{12} V_{02}+e_{12} V_{22} \\
\vdots \\
\frac{\partial V_{1, N}}{\partial \tau}=a_{1, N} V_{1, N-1}+b_{1, N} V_{1, N}+c_{1, N} V_{1, N+1}+d_{1, N} V_{0, N}+e_{1, N} V_{2, N}
\end{gathered}
$$

This pattern continues until when $i=M$, running through $j=1, \ldots N$. Therefore we have a system of $M N$ unknowns. Create a square matrix $M N$ to contain the coefficients of the unknowns and a column vector $f$ of $M N$ rows for the known values at the boundary. For the boundary condition $y=$ $y_{\max }$,

$$
\lim _{y \rightarrow \infty} \frac{\partial V}{\partial y}(x, y, \tau)=0
$$

The point $j=N+1$ is when volatility is at maximum. In a way to avoid having ghost point outside the domain, we use the backward difference to discretize the boundary condition.

$$
\begin{gathered}
\frac{V_{i, N+1}-V_{i, N}}{\Delta y}=0 \\
V_{i, N+1}=V_{i, N}
\end{gathered}
$$

Hence the unknown at the boundary point $N+1$ can be evaluated at point $N$. For the system of equation above, we 
get a tri diagonal block square matrix of size $M \times M$. Each block contains a square matrix of size $N \times N$,

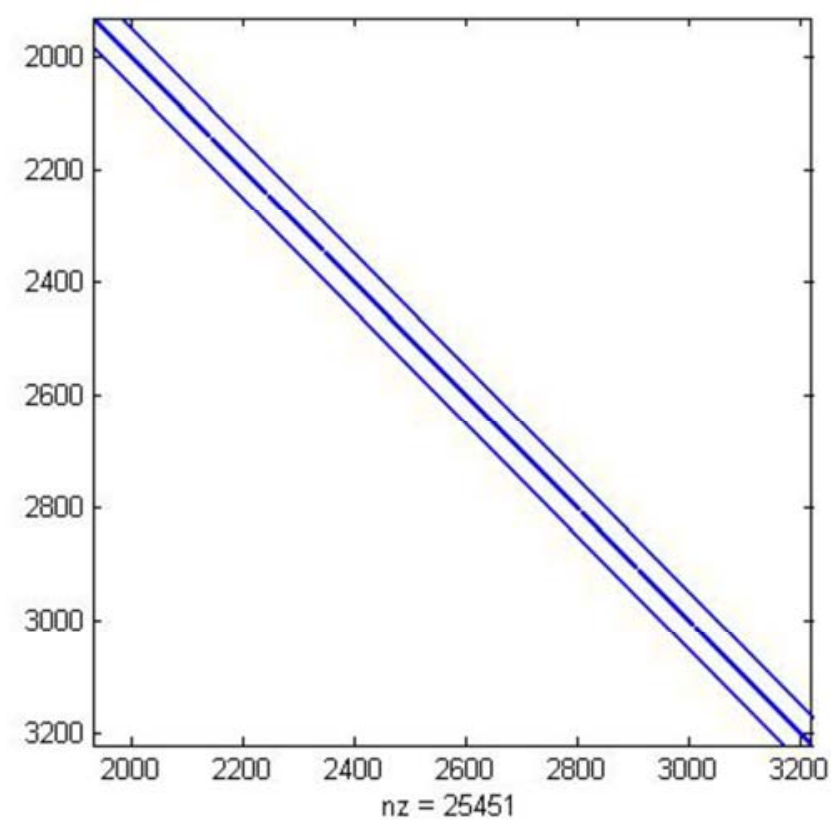

Figure 1. The block tridiagonal matrix using Crank-Nicolson FDM.

The column vector containing the known boundary points and the vector containing the initial condition are given below

$$
f=\left(\begin{array}{c}
a_{00} V_{10}+d_{11} V_{01} \\
d_{12} V_{02} \\
\vdots \\
a_{21} V_{20} \\
0 \\
\vdots \\
a_{31} V_{30} \\
0 \\
\vdots \\
a_{M 1} V_{M 0} \\
0 \\
\vdots
\end{array}\right)\left(\begin{array}{c}
\max (K-x(1), 0) \\
\vdots \\
\max (K-x(2), 0) \\
\vdots \\
\max (K-x(3), 0) \\
\vdots \\
\max (K-x(4), 0) \\
\vdots \\
\max (K-x(M-1), 0) \\
\vdots \\
\max (K-x(M), 0) \\
\vdots
\end{array}\right)
$$

After discretizing the space, $A V$ in (20) becomes $A V=$ $Z V+f$ where $f$ contains the boundary conditions.

Crank-Nicolson FDM is said to be flexible with respect to the choice of time and this can be done in such a way to match cash-flow dates exactly near the option's maturity date [2]. The unconditionally stability of the scheme implies that there is no limitation to the choice of time step $\Delta t$. After the space discretization, the evolved ordinary differential equation is given by

$$
\frac{d V}{d \tau}=A V
$$

We make use of the forward difference formula for the derivative with respect to time

$$
\frac{d V}{d \tau}=\frac{V^{s+1}-V^{s}}{\Delta \tau}
$$

$$
V^{s+1}=(\mathbb{I}-\Delta \tau \theta Z)^{-1}\left((\mathbb{I}+\Delta \tau(1-\theta) Z) V^{s}+\left(\Delta \tau \theta f^{s+1}+\Delta \tau(1-\theta) f^{s}\right)\right)
$$

For $s=\Delta \tau \ldots T$ and II is an identity square matrix of size $M N$.

\subsection{Alternating Direction Implicit (ADI) Scheme}

In the case of ADI spatial discretization, $\mathrm{AV}$ is split into two $A V=A_{1} V+A_{2} V$ It should be noted that AV would have been decomposed into three parts if there was correlation between the stock and volatility. The expression (14) becomes

$$
\begin{array}{cc}
\frac{\partial V}{\partial \tau}=\sum_{n=1}^{2} A_{n} V & Y_{0}=V^{0}+\Delta \tau F\left(t^{s-1}, V^{s-1}\right) \\
Y_{n}=Y_{n-1}+\theta \Delta \tau\left(F_{n}\left(t^{s}, Y_{n}\right)-F_{n}\left(t^{s-1}, V^{s-1}\right)\right) & \text { for }(n=1,2) \\
Y_{2}=V^{s} &
\end{array}
$$

The scheme is given as:
In a way to achieving an efficient numerical solution, it is best to make use of an effective time discretization scheme [4]. Douglas scheme is considered here. The discretized space (ordinary differential equation) can be written as

$$
\begin{gathered}
\frac{d V}{d \tau}=F(t, V(t)), 0 \leq t \leq T \cdot V(0)=V^{0} \\
F(t, V(t))=\sum_{n=1}^{2} Z_{n} V+f_{n}(t)
\end{gathered}
$$

The procedure (29) is repeated for $s \in[0 ; T]$, the scheme is simplified using (27) as follows

$$
\begin{gathered}
Y_{0}=\left(\mathbb{I}+\Delta \tau \sum_{n=1}^{2} Z_{n}\right) V^{s-1}+\Delta \tau \sum_{n=1}^{2} f_{n} t^{s-1} \\
Y_{1}=\left(\mathbb{I}-\theta \Delta \tau Z_{1}\right)^{-1}\left[Y_{0}-\theta \Delta \tau Z_{1} V^{s-1}+\theta \Delta \tau\left(f_{1} t^{s}-f_{1} t^{s-1}\right)\right] \\
Y_{2}=\left(\mathbb{I}-\theta \Delta \tau Z_{2}\right)^{-1}\left[Y_{0}-\theta \Delta \tau Z_{2} V^{s-1}+\theta \Delta \tau\left(f_{2} t^{s}-f_{2} t^{s-1}\right)\right]
\end{gathered}
$$


And hence after the time has run through maturity $(s=T)$, we have $Y_{2}=V^{S}=V^{T}$ which is the required value of the option for each discretized stock prices and volatility.

\section{ComparisonofResults}

The numerical solutions to be discussed in this section were obtained using MATLAB programming language. Maturity time is given in years and prices is Naira, unless otherwise stated the grids used are

$$
\begin{gathered}
M=100 \text { (internal nodes on } \mathrm{x} \text {-axis) } \\
x_{\max }=200 \text { (maximum stock price), } \\
N=50 \text { (internal nodes on y-axis), } \\
y_{\max }=1 \text { (maximum volatility) } \\
L=100 \text { (number of time step), }
\end{gathered}
$$

The option value surface is given below using the following set of parameters $K=100 ; r=0: 1 ; \theta=$ $0: 5 ; \lambda=0: 5 ; k=0: 4 ; T=1$.

The point when volatility is at minimum, the option value is equal to the pay-off. When at maximum, volatility is not used in hedging the stock and hence the option value decreases for stock prices that are far from the strike price.

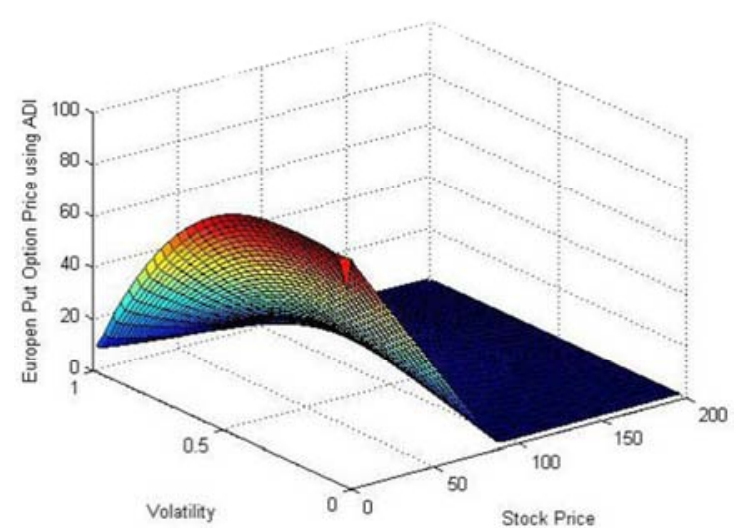

Figure 2. ADI Scheme.

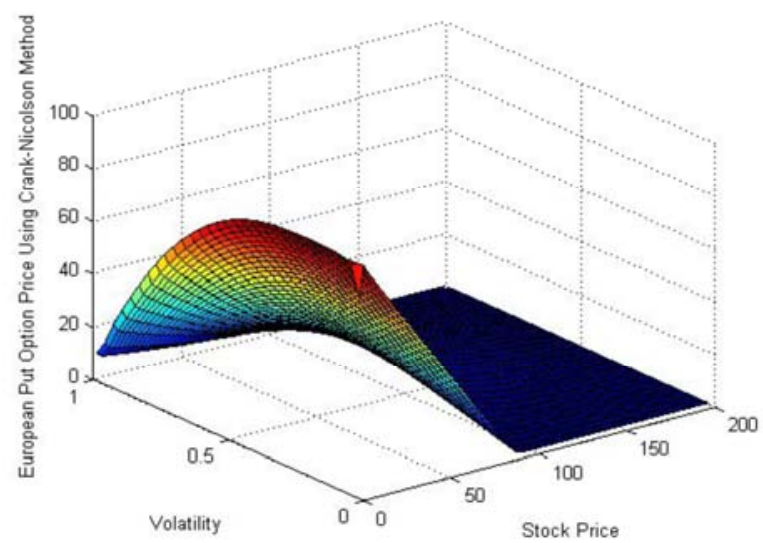

Figure 3. Crank-Nicolson Scheme.

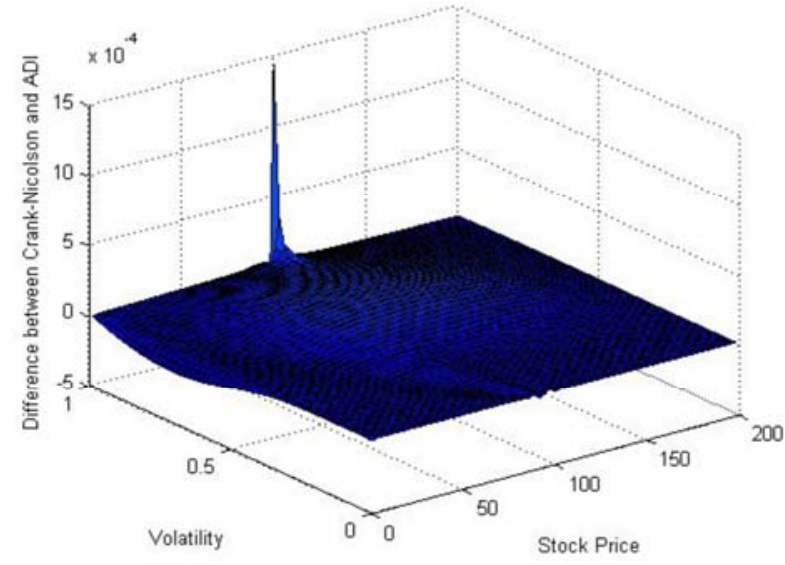

Figure 4. Timestep $L=100$.

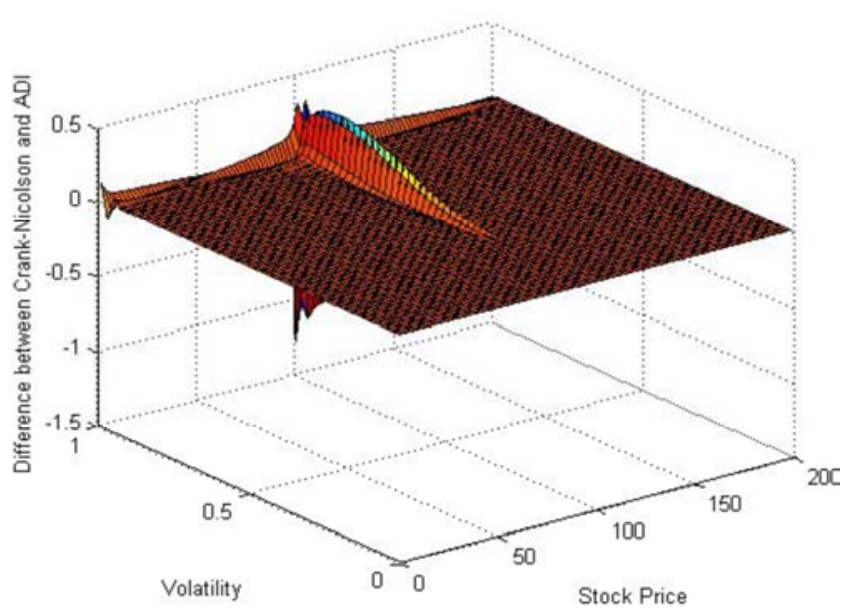

Figure 5. Timestep $L=30$.

Table 1. Option value (ADI) increasing as volatilityincreases.

\begin{tabular}{llll}
\hline \multirow{2}{*}{ StockPrice } & OptionPrice & & \\
\cline { 2 - 4 } & Volatility $=\mathbf{0 . 0 2}$ & Volatility $=\mathbf{0 . 3 2}$ & Volatility $=\mathbf{0 . 4 2}$ \\
\hline 70 & 25.1190248419 & 25.9165682092 & 26.8692944300 \\
80 & 10.4709012713 & 16.4209502722 & 18.6628103500 \\
90 & 1.260192727 & 11.2037237320 & 13.9250683200 \\
100 & 0 & 6.4305729914 & 8.9777769026 \\
122 & $5.6221 \times 10^{-9}$ & 1.7072746508 & 3.4973606134 \\
130 & $8.5996 \times 10^{-8}$ & 2.2256777198 & 4.1879096405 \\
\hline
\end{tabular}

Table 2. Computationa ltime for the two numerical scheme at different grids.

\begin{tabular}{lll}
\hline Grid & ADI & Crank-Nicolson \\
\hline$(80,32,16)$ & 0.8438 & 1.0156 \\
$(100,50,100)$ & 3.2188 & 10.9688 \\
$(160,64,32)$ & 3.5938 & 8.8906 \\
\hline
\end{tabular}

We compare prices relative to Black-Scholes Model. We make the comparison using the following parameters $K=100 ; r=0.1 ; \theta=$ $0.5 ; \lambda=0.5 ; k=0.4 ; T=\frac{1}{2}$ 
Table 3. European put option value in GBM stochastic volatility model relative to Black-Scholes model.

\begin{tabular}{lllll}
\hline Moneyness & StockPrice & Volatility & Black-Schole & ADI \\
\hline Deeply in & 50 & 0.02 & 45.1229424501 & 45.1190248416 \\
In & 70 & 0.06 & 25.1229424500 & 25.1228574753 \\
At & 100 & 0.02 & $6.998869 \times 10^{-5}$ & 0 \\
At & 100 & 0.32 & 6.56234593 & 6.4305767608 \\
Out & 114 & 0.08 & 0 & 0.0049215177 \\
Deeply Out & 122 & 1 & 0 & 0.7216412051 \\
\hline
\end{tabular}

\section{Conclusions}

The model (1)-(2) which is a GBM stochastic volatility was used in pricing European put option numerically, due to the unattainable closed form solution of the model. It was shown that Option value in a GBM stochastic volatility model is an increasing function of volatility i.e.it increases as thevolatilityincreases. Irrespective of the moneyness of the option, this can be seen in Table 1. Option value reduces when time to maturity is long (i.e. the premium paid to the option writer is small), thereby profiting the option holder. ADI finite difference method is more efficient because it takes less time to compute and the structure of the system of equation in form of matrix is not as complex as that of Crank-Nicolson. In the absence of market price of volatility risk, the value of an option increases.

\section{References}

[1] K. Ajayi and T. O Ogunlade. Global Journal of Computer Science and Technology 11(10) pp 5-10, 2011.

[2] M. Bellalah. Derivatives, Risk Management \& Value. World Scientific Publishing Co. Pte. Ltd., 2010.

[3] D. J. Duffy. Finite Difference Methods in Financial Engineering A Partial Differential Equation Approach. John Wiley \& Sons, Ltd, 2006.
[4] K. I. Houtand S. Foulon. ADI Finite Difference Schemes for Option Pricing in the Heston Model With Correlation. International Journal of Numerical Analysis and Modeling, 7:2303-320, 2010.

[5] J. Hull and A. White. Pricing of Options on Assets with Stochastic Volatilities. The Journal of Finance, 42:281-300, 1987.

[6] S. Ikonen and J. Toivanen. Operator Splitting Methods for American Options with Stochastic Volatility. European Congress on Computational Methods in Applied Sciences and Engineering, 2004.

[7] M. Musiela and M. Rutkowski. Martingale Methods in Financial Modelling. Springer, 2004.

[8] C. R. Nwozo and S. E. Fadugba. On Stochastic Volatility in the Valuation of European Options. BritishJournal of Mathematics \& Computer Science, 5:104-127, 2014.

[9] F. D. Rouah. The Heston Model and Its Extensions in Matlab and C\#. John Wiley \& Sons, Inc., 2013.

[10] S. E. Shreve. Stochastic Calculus for Finance II: Continuoustime models, volume 11. Springer Science\& Business Media, 2004.

[11] U. F. Wiersema. Brownian Motion Calculus. John Wiley \& Sons Ltd, 2008.

[12] S.-P. Zhu and W.-T. Chen. A New Analytical Approximation for European Puts with Stochastic Volatility. Applied Mathematics Letters., page 23 (2010) 687-692, 2010. 